White paper submitted to the Planetary Science and Astrobiology Decadal Survey 2023-2032

Category: Interdisciplinary/Other

\title{
THE NEED FOR RECOMMENDATIONS IN SUPPORT OF PLANETARY BODIES CARTOGRAPHIC COORDINATES AND ROTATIONAL ELEMENTS STANDARDS
}

\author{
Lead Author:
}

Flora Paganelli, Green Bank Observatory, Green Bank, WV, USA, fpaganel@nrao.edu, 518418-9155

Co-Authors:

Brent A. Archinal, U.S. Geological Survey, Flagstaff, AZ, USA;

Charles H. Acton, Jet Propulsion Laboratory, California Institute of Technology, Pasadena, CA, USA;

Albert Conrad, Large Binocular Telescope Observatory, Tucson, AZ, USA;

Tom C. Duxbury, George Mason University, Fairfax, VA, USA;

Daniel Hestroffer, IMCCE, Observatoire de Paris, Université PSL, CNRS, Sorbonne Université, Univ. Lille, Paris, France;

James L. Hilton, U.S. Naval Observatory, Washington, DC, USA;

Laurent Jorda, Laboratoire d'Astrophysique de Marseille, Marseille, France;

Randolph. L. Kirk, U.S. Geological Survey, Flagstaff, AZ, USA;

Sergei A. Klioner, Lohrmann Observatory, Technische Universität Dresden, Dresden, Germany; Jean-Luc Margot, University of California, Los Angeles, CA, USA;

Juergen Oberst, DLR Berlin Adlershof, Berlin, Germany;

Jinsong Ping, National Astronomical Observatories of CAS, Beijing, China;

Paul K. Seidelmann, University of Virginia, Charlottesville, VA, USA;

David J. Tholen, University of Hawaii, Honolulu, HI, USA;

Iwan P. Williams, Queen Mary University of London, London, UK.

Endorsers:

Amber Bonsall, Green Bank Observatory, abonsall@nrao.edu;

Mitchell K. Gordon, SETI Institute, mgordon@seti.org;

David P. Mayer, U.S. Geological Survey, Astrogeology Science Center, dpmayer@usgs.gov;

Andrea Nass, German Aerospace Center (DLR), Berlin, Germany, andrea.nass@dlr.de;

Louise M. Prockter, Johns Hopkins University Applied Physics Laboratory,

louise.prockter@jhuapl.edu;

Jani Radebaugh, Brigham Young University, janirad@byu.edu;

Sarah S. Sutton, University of Arizona, ssutton@lpl.arizona.edu;

Jill Tarter, SETI Institute (retired), tarter@seti.org. 


\section{EXECUTIVE SUMMARY}

This paper considers how, since 1976, the International Astronomical Union Working Group on Cartographic Coordinates and Rotational Elements (WGCCRE) has made recommendations regarding coordinate systems and rotational element standards for planetary bodies that are needed for mapping and the planning, execution, and interpretation of observations. We recommend that the Planetary Science Decadal and Astrobiology Decadal Survey committee and panels (hereafter, the "Survey") should endorse the value of cartographic standards as provided by the WGCCRE, suggest the collection of community input to improve the working group's services, and describe how increased support would enable key improvements in order to achieve the benefits of enhanced return from planetary science data.

\section{MOTIVATION AND STATE OF THE ART}

In 1976, the International Astronomical Union (IAU) established the Working Group on Cartographic Coordinates and Rotational Elements (WGCCRE) to recommend both fundamental principles and specific parameter values pertaining to planetary coordinates and mapping. The WGCCRE is a volunteer organization, though the current chair receives fractional-time support from NASA to coordinate its activities. The group does not conduct research on improving planetary reference frames but relies on published results and community input to make its recommendations. It has no enforcement powers and tries to make persuasive recommendations to the international planetary exploration community that reflect the long-term community consensus. Approximately every three years, the group issues a report (e.g., Archinal et al., 2018) containing standards for establishing and updating planetary coordinates and current best values of parameters (including body orientation and shape). These standardized procedures, definitions, and values can be used by the planetary community to support observational and theoretical work and make cartographic products in a consistent way, which facilitates the use and comparison of multiple datasets and thus advances both mission planning and research. Standards for formatting digital cartographic products fall outside the group's purview and are addressed by the NASA Planetary Data System (PDS) and other organizations.

After 44 years of operation, the WGCCRE is considering how its practices and interactions with other organizations might be improved and is preparing to gather community input on these topics (Archinal et al., 2020). As part of this process, we have also come to realize that the WGCCRE has accumulated enough experience and evidence that it would be useful to make recommendations to the Survey on topics related to planetary mapping. This includes specific recommendations related to planetary coordinates and mapping. It also includes a more general finding that further study and collection input from the planetary community would be beneficial to improving the services that the working group currently provides.

Our specific recommendations are based on inquiries we have received, problems that have historically occurred when missions adopted unusual to non-standard/recommended procedures, failures (perhaps at times by the WGCCRE) to obtain sufficient input from the community, and limitations on the ability of the WGCCRE and others to address significant issues due to lack of resources. These situations have caused problems to varying degrees to everyone in the planetary community, including space agencies, missions, instrument teams, data users, and researchers in general. Some specific examples include:

- For many planetary bodies, particularly the Moon and Mars, most datasets have never been well registered (or at least with any measure of accuracy) to each other, making data 
comparison and fusion difficult (Archinal et al., 2016) and lowering the value of datasets, which were obtained at great difficulty and cost.

- The NASA/DLR/ASI Dawn mission initially did not update and register its data to the existing global coordinate system for Vesta, resulting in great (and at times, continued) confusion as to which system such data are in (Archinal et al., 2018, Section 4).

- Articles in refereed journals have claimed to use a coordinate system that follows IAU recommendations when they do not.

- Various missions have chosen to use planetocentric coordinates to register data of bodies such as Mercury and the Galilean Satellites. Use of planetographic coordinates for all previous ground-based observations and mission data results in issues in using such data in planetocentric coordinates.

- Questions exist about an offset in longitude between the previous and currently recommended Mars coordinate system (Archinal et al., 2020, Section 5). The WGCCRE does not currently have enough personnel to address that issue.

- Similarly, substantial new lunar laser-ranging and lunar gravity data could be used to improve the definition of the coordinate system for the Moon, but who could or will have time to undertake that work is not clear.

These issues have been discussed within the WGCCRE and other groups for some time. Some have come up recently and require further discussion by the working group and the community. We therefore describe the WGCCRE's operations and involvement with other organizations. In the remaining two sections of this paper, we present recommendations that the Survey can make regarding coordinate and mapping standards. Section 2 includes specific recommendations to the community on actions that we believe should be taken now. Section 3 addresses the future of the WGCCRE and the work it currently performs. The main question is whether to continue the existing WG (perhaps with some expansion), or whether some new method of operation or structure would be more beneficial to NASA and the international planetary community. The WGCCRE feels more input is needed from the planetary science community in order to finalize what should be done on these topics. It is possible that obtaining adequate input will be difficult, but we feel it is critical to make sure the community is aware of these issues and has a chance to respond, before making further decisions. We have seen many cases of small groups, such as a limited number of authors, instrument teams, and missions - and arguably WGCCRE itself on occasion - making key decisions where community input should have been sought and would have prevented some of the confusion regarding planetary coordinates and mapping that we now face.

Improving communication and coordination with the community and a range of other organizations is also key to future WGCCRE activities. Questions that remain open are how can the group better communicate the value of its activities and of adherence to mapping standards to the lunar and planetary community? Can the WGCCRE interact more closely with other professional societies, space agencies, and working groups to foster international agreement on consistent use of cartographic standards and obtain the feedback needed to establish community consensus?

Our discussion of these topics is naturally focused on the role and operations of NASA, given the function of the Survey, but many of our conclusions and recommendations apply to all space 
agencies. NASA can play a role in encouraging the cooperation of those agencies in building a global approach to mapping standards.

There are many other established international, national, and space agency groups whose terms of reference overlap with the WGCCRE, including several NASA working groups, multiple IAU Commissions, and groups within professional societies such as the International Society of Planetary Remote Sensing (ISPRS), International Cartographic Association, Committee on Space Research, and the International Association of Geodesy. The WGCCRE has opened discussions with some of these groups, but its current resources are probably insufficient to sustain the level of effort needed to implement consistent policies on mapping standards. Two especially important groups, the NASA Mars Geodesy and Cartography Working Group (MGCWG) and Lunar Geodesy and Cartography Working Group (LGCWG) are no longer active despite an ongoing need for their efforts as described below. Similarly, the WGCCRE is organizing opportunities for discussion of mapping issues at upcoming meetings (e.g., through existing or WG-organized Town Hall events), but resources limit the number of such events that can be supported.

\section{RECOMMENDATIONS FOR ACTION NOW}

Here are specific recommendations for action, derived from the current experience of the WGCCRE and in discussions between members of the WGCCRE and the planetary science community. We consider these items to be important, if not critical, to NASA and other space agencies in order to obtain the best possible and most efficient use of planetary geospatial data.

2.1 Support for the WGCCRE. As the volume and complexity of planetary datasets and the number of mapped bodies increase, the WGCCRE has had great difficulty as a mostly volunteer organization in keeping up with addressing the resulting issues and questions regarding planetary coordinates and rotational elements, and other high-level cartographic standards. Whether reorganization of this work would be beneficial is discussed in the next section. Here, we point out that support of the existing WG would result in significant benefits toward addressing the issues just discussed. Dedicated funding would allow WG members to meet and work with the other organizations. An important component of this work would be to ask for, evaluate, interpret, and act on feedback received from the community towards establishing a consensus that would foster consistent use of cartographic and rotational standards. This can only be achieved through effective and expanded interaction of the WGCCRE with other professional societies, space agencies, and working groups closely linked to the type of work of the WGCCRE.

2.2 NASA Missions and Instrument Teams. Missions and instrument teams, before and after selection, should follow cartographic and rotational standards best practices. We strongly recommend that NASA work directly with the WGCCRE (and related groups) to require compliance to such standards. Similar cooperation by other space agencies would be equally beneficial and could be encouraged by NASA. If the guidelines are not implemented, teams should be required to explain their rationale for departing from such standards and discuss needed changes or improvements with the WGCCRE or other relevant standards groups. Such formal requirements would constitute new policy on the part of the agencies. One way to simplify compliance with these requirements is the use of the "SPICE" system (Acton, 1996; Acton et al., 2017). SPICE consists of software and data files of observation geometry ("kernels") from the NASA Navigation and Ancillary Information Facility (NAIF) that can be 
used for both planning and analyzing spacecraft observations. The NAIF team already works with multiple space agencies and has established training in support of NASA, ESA, and other space agencies' exploration activities, exposing generations of scientists and engineers to coordinate standards. Direct coordination between the WGCCRE and NAIF can leverage this service to promote awareness of related standards such as creating and updating coordinate systems and should be explicitly supported by NASA. At a minimum, cartographic and rotational standards best practices should be followed, science data providers should be required to include appropriate observation geometry metadata along with their science data archives, and peer reviewers should make a reasonable effort to check such metadata before accepting an archive submittal.

2.3 Journals. NASA (and other space agencies) should support the use of cartographic and rotational standards best practices for journal publications. NASA, along with the WGCCRE, should encourage journal editors to establish formal policies requiring (or at least encouraging) compliance with community-accepted cartographic standards. Such policies would have avoided substantial confusion in several recent cases in which authors did not follow WGCCRE recommendations and users were forced to convert either existing or new datasets between conflicting coordinate systems in order to work with them. Existing journal requirements (e.g., for meteorite designations; Heck et al., 2019) provide models for such policies. Again, without additional financial support, it would be difficult or impossible for the WGCCRE to work with journal editors and when asked to help review papers or advise on such issues.

2.4 NASA Geodesy/Cartography Working Groups. The Mars and Lunar Geodesy and Cartography Working Groups (MGCWG and LGCWG) chartered by NASA, with the aim of supporting U.S. missions' coordination for spatial accuracy and consistency of map products, resolving discrepancies, and helping to develop observation plans, were active in the 1990s to early 2000s but have since become inactive. The groups have been responsible for profound and fundamental results, including helping to verify Mars landing site mapping products and defining the coordinate systems for the Mars (Duxbury et al., 2002) and the Moon LRO Project and LGCWG (2008). In 2018-2019, there was also an attempt to create a related International Phobos-Deimos Data Working Group to address similar issues for those bodies. Though NASA chartered, the Mars and lunar groups had significant non-U.S. participation and considered international input. As a consequence, their informal findings were generally persuasive to agencies and mission teams of other nations. Given the broader scope of these groups, compared to the focus of the WGCCRE on cartographic and rotational standards, these groups should be revived. However they are constituted, the group(s) would provide support to agencies, missions, instrument teams, and the WGCCRE. They could also assist in the development of planetary spatial data infrastructures (PSDIs) as discussed in Section 2.6. Effective operation of these groups would likely require partial financial support for a modest number of members to analyze data products, formulate detailed recommendations, and chair and operate these groups, even if most members work as volunteers or rely on other funding sources. The need for these groups is not hypothetical. As noted above, questions exist about an offset in longitude between the previous and currently recommended Mars coordinate system that should be resolved soon, and the lunar coordinate system needs updating based on new data. The WGCCRE relied on these groups previously, and currently does not have enough personnel to address these issues in a timely way, so confusion about all Mars and lunar mapping products, including critical landing site mapping products on both bodies, may result. 
2.5 Exoplanets community. The Exoplanet Science Strategy (NAS, 2018) describes exoplanet science as an "avalanche of unexpected discoveries." This exemplifies growing awareness within the space science community that scientific progress would benefit from pathways and resources for research coordination among disciplines (see white paper by Mandt et al., 2020). We recommend that here too, a new "Geodesy and Cartography Working Group" of some sort be created to establish initial recommendations regarding planetary coordinates and rotational elements and other high-level cartographic standards. The WGCCRE could facilitate such coordination by working with both the Solar System and exoplanet communities but again does not currently have the resources to address such issues on its own. Though no extrasolar bodies have currently mappable surface features, some may in the future, and sizes and rotation rates for many are likely to become known in the nearer term. Researchers in both fields will benefit if such information is presented according to consistent definitions and can thus be compared easily.

2.6 Planetary Defense Community. The planetary defense community would benefit from knowing about and following the cartographic and rotational standards already in use by planetary scientists. Conversely, planetary defense research is likely to yield models and data of interest to planetary scientists provided they are in understandable forms. Cartographic and rotational standards affect various models commonly used for planetary defense as part of the National Near-Earth Object Preparedness Plan, such as orbital models, threat identification, deflection and disruption models, and models of the effects and risks of airbursts and impacts. These issues are described in the white paper by Stickle et al. (2020) which emphasizes the need for coordinated support - including cartographic support-from the planetary community. It goes almost without saying that confusion over coordinate systems could have much more serious consequences in the context of a future planetary defense operation than in recent examples that merely imposed obstacles to planetary research. We therefore recommend that here too a Geodesy and Cartography Working Group or similar organization be created and supported in order to consider these issues, and - like the previous Mars and lunar groups - make recommendations to the WGCCRE.

2.7 PSDI Development. The functions of the WGCCRE fit clearly into the evolving concept of developing a planetary spatial data infrastructure (PSDI) with international scope (Laura et al., 2017), whether body-by-body or as a single infrastructure for the whole Solar System. The WGCCRE efforts are already cited as the likely foundational standards portion of such work (ibid., Section 3.3), but other PSDI topics are also relevant, such as policies (e.g., requirements and needs of space agencies), people (workforce planning to maintain sufficiently qualified and experienced staff to do the work), access networks (to obtain planetary data in useful formats), and data, in the form of reference frame, elevation, and mosaic foundational data products, and framework products for other data types. Such efforts would be in line with the Mapping and PSDI Roadmap by the NASA MAPSIT (MAPSIT, 2019) and input to the Survey (see the white paper by MAPSIT, 2020). The Survey should recommend that PSDIs be created, following WGCCRE recommendations on reference frames.

2.8 Controlled Products. Routine generation of controlled products has been recommended by several authors, including but not limited to the WGCCRE (Archinal et al., 2018, Section 9; Committee on the Planetary Science Decadal Survey, 2011, p. 5-16; International Space Development Conference, 2015; Archinal et al., 2016; Laura et al., 2017; MAPSIT, 2019, Finding I). The generation of controlled products also places an extra burden (though still a 
miniscule fraction of total investigation cost that substantially enhances the overall scientific return) on the teams charged with data analysis. The work by the WGCCRE and others to establish and recommend coordinate systems and rotational elements for Solar System bodies is for nothing if planetary geospatial data are not controlled and properly registered into those systems. Such products also are needed and serve as the foundational data products of any PSDI. We believe the value of controlled products for scientific research and mission use is sufficiently great that NASA should require their production and consider committing to providing teams with the necessary resources.

\section{LONG-TERM EFFORTS AND RECOMMENDATIONS FOR NASA}

What is the future of this type of work and the WGCCRE? Improvements to the existing WGCCRE have been discussed, but it is worthwhile to consider more fundamental restructuring. One model would be to have geodesy/cartography working groups assist or take over the efforts of the WGCCRE. The International Earth Rotation and Reference Systems (IERS) also provides an alternative model.

3.1 New Organizational Structures for Promoting Cartographic and Rotational Standards. Geodesy/cartography working groups, chartered by NASA in the past, provide one possible model for supporting WGCCRE activities through partial funding of the members. The IERS serves the terrestrial and astronomical communities. That organization provides a model of how similar functions are carried out that could be used to augment or even replace the role of the WGCCRE in providing cartographic standards support to the planetary community. It performs the service of establishing coordinate systems and frames for the Earth and inertial space and the connection between the two (Earth rotation), much as the WGCCRE does for all other Solar System bodies. Though the amount of work done by the IERS may be greater, that of the WGCCRE is at least as complex, as a result of the many bodies being considered. The IERS includes a Directing Board that oversees policy and operation and the development of standards and operational components that perform the routine work of the organization. Based on this model, operational components for planetary cartographic and rotational standards oversight could potentially be handled by replacing the WGCCRE with an oversight board (perhaps an IAU Commission). Operationally, this model would employ dedicated staff at various institutions, possibly supported by various space agencies.

Whether it is appropriate to adopt these approaches needs to be evaluated through comparison of increased benefits vs. costs involved. In the near term, NASA can encourage the development of community consensus on the value of these possible organizational changes. In the longer term, NASA might need to assist by chartering one or more new organizations and at least partially fund their operations. NASA could also play a major role by negotiating with other space agencies to support these organizations through policy and financial support.

We therefore recommend that NASA give serious consideration to the creation of a planetary cartographic and rotational standards service, in close consultation with the IAU and the partner space agencies that would be called to recognize and contribute financial support to such a body.

\section{ACKNOWLEDGEMENTS}

A portion of this research was carried out at the Jet Propulsion Laboratory, California Institute of Technology, under a contract with the National Aeronautics and Space Administration (80NM0018D0004). Funding for B. Archinal has been provided via a National Aeronautics and Space Administration-U.S. Geological Survey Interagency Agreement on Planetary Spatial 
Data Infrastructure. Green Bank Observatory is supported by the National Science Foundation and is operated by Associated Universities, Inc. F. Paganelli carried out a portion of this research under SETI Institute funding.

\section{REFERENCES}

Acton, C. H., 1996. Ancillary data services of NASA's Navigation and Ancillary Information Facility, PSS 44, 1, 65-70, https://doi.org/10.1016/0032-0633(95)00107-7

Acton, C. et al., 2017. A look toward the future in the handling of space science mission geometry. PSS, https://doi.org/10.1016/j.pss.2017.02.013

Archinal, B. A. et al., 2016. Registering Planetary Datasets for Data Fusion: A "Force Multiplier" for Planetary Science, LPS 47, \#2377.

Archinal, B. A. et al., 2018. Report of the IAU WGCCRE: 2015, CMDA, 130:22, doi.org/10.1007/s10569-017-9805-5

Archinal, B. and the IAU WGCCRE, 2020. Coordination of Planetary Coordinate System Recommendations by the IAU Working Group on Cartographic Coordinates and Rotational Elements - 2020 Status and Future. XXIV ISPRS Congress 2020, TCIII-II: Planetary Remote Sensing and Mapping, ID:326. Nice, France. https://www.int-arch-photogramm-remote-sensspatial-inf-sci.net/XLIII-B3-2020/1091/2020/

Committee on the Planetary Science Decadal Survey, 2011. Vision and Voyages for Planetary Science in the Decade 2013-2022, The National Academies Press, Washington, D.C.

Duxbury, T. C., et. al., 2002. Mars Geodesy/Cartography Working Group Recommendations on Mars Cartographic Constants and Coordinate Systems, ISPRS, v. 34, part 4.

Heck, P. R., et al., 2019. Best practices for the use of meteorite names in publications, Meteoritics \& Planetary Science, 54, 7, 1397-1400.

International Space Development Conference, 2015. International Lunar Decade Declaration, International Space Development Conference, Toronto, CA. National Space Society, May 25. https://space.nss.org/international-lunar-decade-declaration-unveiled-at-national-space-societyconference/, https://space.nss.org/wp-content/uploads/International-Lunar-Decade-

Declaration.pdf

Laura, J. et al., 2017. Towards a Planetary Spatial Data Infrastructure. ISPRS Int. J. Geo-Inf. 6, 181. http://www.mdpi.com/2220-9964/6/6/181/pdf

LRO Project and LGCWG, 2008. A Standardized Lunar Coordinate System for the Lunar Reconnaissance Orbiter and Lunar Datasets, Version 5, October 1

Mandt et al., 2020. Advancing Space Science Requires NASA Support for Coordination Between the Science Mission Directorate Communities. The Planetary Science and Astrobiology Decadal Survey 2023-2032.

Mapping and Planetary Spatial Infrastructure Team (MAPSIT), 2019. Mapping and PSDI Roadmap 2019-2023, June 19. https://www.lpi.usra.edu/mapsit/roadmap/MAPSIT-Roadmap2019-06-19.pdf

Mapping and Planetary Spatial Infrastructure Team (MAPSIT), 2020. Maximizing the Value of Solar System Data through Planetary Spatial Data Infrastructures. The Planetary Science and Astrobiology Decadal Survey 2023-2032. https://www.lpi.usra.edu/mapsit/roadmap/MAPSITRoadmap-2019-06-19.pdf

National Academies of Sciences (NAS), Engineering, and Medicine, 2018. Exoplanet Science Strategy. Washington, DC: The National Academies Press. https://doi.org/10.17226/25187

Stickle et al., 2020. The Need For a Well-defined Modeling Pipeline For Planetary Defense. The Planetary Science and Astrobiology Decadal Survey 2023-2032. 